\title{
Modulation of twin-emitter semiconductor lasers beyond the frequency of relaxation oscillations
}

\author{
Geoffrey A. Wilson and Richard K. DeFreez \\ Oregon Graduate Institute of Science and Technology, Department of Applied Physics and Electrical Engineering, \\ 19600 N.W. Von Neumann Drive, Beaverton, OR 97006-1999, USA
}

and

Herbert G. Winful

Department of Electrical Engineering and Computer Science, University of Michigan, Ann Arbor, MI 48109-2122, USA

Received 5 September 1990; revised manuscript received 14 December 1990

\begin{abstract}
Self-modulation above the relaxation oscillation frequency has been previously observed in a phased-array semiconductor lasers. Rate equations for a twin-emitter phased-array semiconductor laser are used to show that active optical modulation above the relaxation oscillation frequency can be achieved by introducing a $180^{\circ}$ relative phase shift in the emitter current modulation.
\end{abstract}

\section{Introduction}

Semiconductor lasers are important as transmitters in high-speed optical communications, largely because their optical output can be modulated directly by varying the injection current. Until now, however, modulation bandwidths have been rather inconveniently limited. The optical output of a single-mode semiconductor laser cannot be effectively modulated beyond its relaxation oscillation frequency, usually less than $10 \mathrm{GHz}$. On the other hand, a multi-longitudinal-mode device can be modulated near the frequency associated with the mode spacing [1]. This is usually greater than $100 \mathrm{GHz}$, which, because of high-frequency parasitics, is beyond the range of present semiconductor laser driver technology. A semiconductor laser that could be modulated at frequencies intermediate to the relaxation oscillation and longitudinal mode spacing frequencies would be highly desirable.

Phased-array semiconductor lasers have been observed to self-modulate during $\mathrm{cw}$ operation at $\mathrm{K}$ band frequencies $(12.5-40 \mathrm{GHz})$, considerably beyond the relaxation oscillation frequency [2-4]. This behavior can be considered as an undamped and essentially passive transfer of photons among the emitters due to their overlapping optical fields [5]. The twin-emitter semiconductor laser phased-array has been studied extensively [6]. In particular, it has been suggested that multi-gigahertz instabilities associated with cycling among a continuum of nonstable near-field distributions might be effectively exploited in twin-emitter arrays, although no modulation scheme was proposed [7]. In this paper we use a simple model of a twin-emitter array to explore the possibility of driving the periodic transfer of photons between the emitters by modulating the injection current to the emitters. Out-of-phase modulation of the emitters is shown to be essential for effective modulation beyond the relaxation oscillation frequency, opening new possibilities for highspeed optical communications and other applications.

\section{Twin-emitter phased-array model}

The twin emitter is the simplest semiconductor 
laser array, consisting of two identical emitters placed side by side in the junction plane. The goal of this section is to model the response of a twin-emitter array to injection current modulation as a function of the frequency and the relative phase of modulation of the emitters.

A rate equation model has been presented [8] that considers the emitters as oscillators whose electric fields are coupled through a real parameter $\eta$. The frequency of unstable oscillations was shown to be proportional to $\eta$. That model has been extended [5] to include complex coupling by allowing $\eta \rightarrow \eta_{R}+$ $\mathrm{i} \eta_{\mathrm{I}}$, where $\eta_{\mathrm{I}}$ can be associated with lateral mode discrimination [9]. Coupled-mode theory can be used to estimate $\eta_{\mathrm{R}}$ and $\eta_{\mathrm{l}}$, or they can be measured from the emission spectrum of a twin-stripe array [3].

The rate equations used are

$$
\begin{aligned}
\dot{X}_{1} & =Z_{1} X_{1}-\eta_{\mathrm{R}} X_{2} \sin \theta-\eta_{1} X_{2} \cos \theta \\
\dot{X}_{2} & =Z_{2} X_{2}+\eta_{\mathrm{R}} X_{1} \sin \theta-\eta_{\mathrm{I}} X_{1} \cos \theta \\
\dot{\theta} & =-\alpha\left(Z_{2}-Z_{1}\right)+\eta_{\mathrm{R}}\left(\frac{X_{1}}{X_{2}}-\frac{X_{2}}{X_{1}}\right) \cos \theta \\
& +\eta_{\mathrm{I}}\left(\frac{X_{1}}{X_{2}}+\frac{X_{2}}{X_{1}}\right) \sin \theta \\
\dot{Z}_{1} & =\frac{p^{(0)}+p^{(1)}(t)-Z_{1}-\left(1+2 Z_{1}\right) X_{1}^{2}}{T}, \\
\dot{Z}_{2} & =\frac{p^{(0)}+L p^{(1)}(t)-Z_{2}-\left(1+2 Z_{2}\right) X_{2}^{2}}{T},
\end{aligned}
$$

where the dot signifies differentiation with respect to time normalized to the photon lifetime $\tau_{\mathrm{p}}$. The average injection current is represented by $p^{(0)}$ and the modulation by $p^{(1)}$, both appropriately normalized with $p^{(0)}=0$ corresponding to threshold. $L= \pm 1$ in eq. ( $3 \mathrm{~b}$ ) allows the emitters to be modulated in-phase or out-of-phase relative to each other. The normalized electric field and gain for each emitter are represented by $X$ and $Z$, respectively, and $\theta$ denotes the relative phase of the emitter fields. Device parameters include $\alpha$, the linewidth enhancement factor, and $T$, the ratio of the carrier lifetime to the photon lifetime. The quantities appearing in eqs. (1)-(3) have been defined in ref. [8].

The use of rate equations becomes invalid as the modulation frequency approaches the polarization relaxation rate $[10]$. Since the latter is typically $\approx 10^{13} \mathrm{~s}^{-1}$, rate equations are appropriate at $\mathrm{K}$-band modulation frequencies. To emphasize emitter coupling effects, the model is intentionally simple, neglecting single-emitter effects such as spontaneous emission and gain nonlinearity.

Setting $p^{(1)}$ and the left-hand sides of eqs. (1)-(3) to zero yields the equilibrium points of the autonomous (unmodulated) device,

$X_{1}^{(0)}=X_{2}^{(0)}=\left(\frac{p^{(0)}-\eta_{\mathrm{I}} \cos \theta^{(0)}}{1+2 \eta_{\mathrm{I}} \cos \theta^{(0)}}\right)^{1 / 2} \equiv X^{(0)}$,

$\theta^{(0)}=0$ or $\pi$,

$Z_{\mathrm{I}}^{(0)}=Z_{2}^{(0)}=\eta_{\mathrm{I}} \cos \theta^{(0)} \equiv Z^{(0)}$.

The $\theta^{(0)}=0$ and $\theta^{(0)}=\pi$ equilibrium points can be associated with in-phase and out-of-phase lateral modes of the array, respectively. Since the equilibrium modal photon density is proportional to $X^{(0) 2}$, it can be seen from eq. (4a) that $\eta_{1}>0$ corresponds to the out-of-phase mode having a larger equilibrium photon density than the in-phase mode, and thus presumably dominates the emission spectrum. The opposite situation exists if $\eta_{1}<0$. A gain-guided twin emitter has been observed to favor in-phase operation [3], justifying the extension of the model to include complex coupling.

\section{Small-signal responsivity}

To obtain the response of the array to small-signal current modulation, let $p^{(1)}$ and the time-dependent perturbations of the dynamical variables be infinitesimal sinusoids, e.g., $p^{(1)} \rightarrow \operatorname{Re}\left(\tilde{p} \mathrm{e}^{\mathrm{i} \Omega t}\right)$, where $\Omega$ is the angular frequency and $\tilde{p}$ is the complex amplitude of the modulation. Eqs. (1)-(3) can then be linearized by replacing their right-hand sides by the associated jacobian matrix evaluated at an equilibrium point given by eq. (4), multiplied by the column vector of the perturbations of the dynamical variables and added to the column vector of driving terms. The common $\mathrm{e}^{\mathrm{i} \Omega t}$ time dependence is then dropped, and the matrix equation is solved for the dynamical variable perturbations.

For in-phase modulation $(L=1)$ 


$$
\begin{aligned}
& \tilde{X}_{1} / \tilde{p}=\tilde{X}_{2} / \tilde{p} \\
& =\frac{X^{(0)}}{-T \Omega^{2}+\mathrm{i}\left(1+2 X^{(0) 2}\right) \Omega+2 X^{(0) 2}\left(1+2 Z^{(0)}\right)},
\end{aligned}
$$

where $\tilde{X}$ is the complex amplitude of the electric field perturbation. The most interesting feature of eq. (5) is the absence of $\eta_{\mathrm{R}}$. Since $\eta_{\mathrm{R}}$ is proportional to the rate at which photons are exchanged between the emitters, its failure to appear suggests that in-phase modulation may be unable to drive the periodic photon transfer needed for a strong high-frequency response. This is reasonable on physical grounds because identical modulation to the emitters does nothing to drive photon transfer preferentially from one emitter to the other. Indeed, the behavior of a twin-emitter array subjected to in-phase modulation as described by eq. (5) is similar to that of a singleemitter device $[11,12]$, becoming identical as $\eta_{1} \rightarrow 0$.

For out-of-phase modulation $(L=-1)$,

$$
\begin{aligned}
& \tilde{X}_{1} / \tilde{p}=-\tilde{X}_{2} / \tilde{p} \\
& =\frac{X^{(0)}\left[1+\left(2 \mathrm{i} \alpha \eta_{\mathrm{R}} / \Omega^{\prime}\right) \cos \theta^{(0)}\right]}{-T \Omega^{\prime 2}+\mathrm{i} A \Omega^{\prime}+B-\mathrm{i} C / \Omega^{\prime}},
\end{aligned}
$$

where

$$
\begin{aligned}
\Omega^{\prime} & \equiv \Omega+2 \mathrm{i} Z^{(0)} \\
A & \equiv 2 T Z^{(0)}+1+2 X^{(0) 2}, \\
B & \equiv 4 T \eta_{\mathrm{R}}^{2}+2 X^{(0) 2}\left(1+2 Z^{(0)}\right), \\
C & \equiv 4 \eta_{\mathrm{R}}^{2}\left(2 T Z^{(0)}+1+2 X^{(0) 2}\right) \\
& -4 \alpha \eta_{\mathrm{R}} X^{(0) 2}\left(1+2 Z^{(0)}\right) \cos \theta^{(0)} .
\end{aligned}
$$

Note that eq. (6) retains $\eta_{R}$, which suggests that photon transfer can be driven using out-of-phase modulation.

As a final step in obtaining the response of the twinemitter array to small-signal modulation, we can define the normalized response function as the ratio of the average-to-peak variations $(\delta)$ in the normalized cavity photon density $\left(X^{2}\right)$ and the normalized injection current $(p)$,

$$
R(\Omega) \equiv \frac{\delta X^{2}}{\delta p} \approx 2 X^{(0)} \frac{\delta X}{\delta p}=2 X^{(0)}\left|\frac{\tilde{X}}{\tilde{p}}\right|
$$

Similar definitions appear in refs. [11] and [12].

Fig. 1 shows the modeled response of a twin-em-

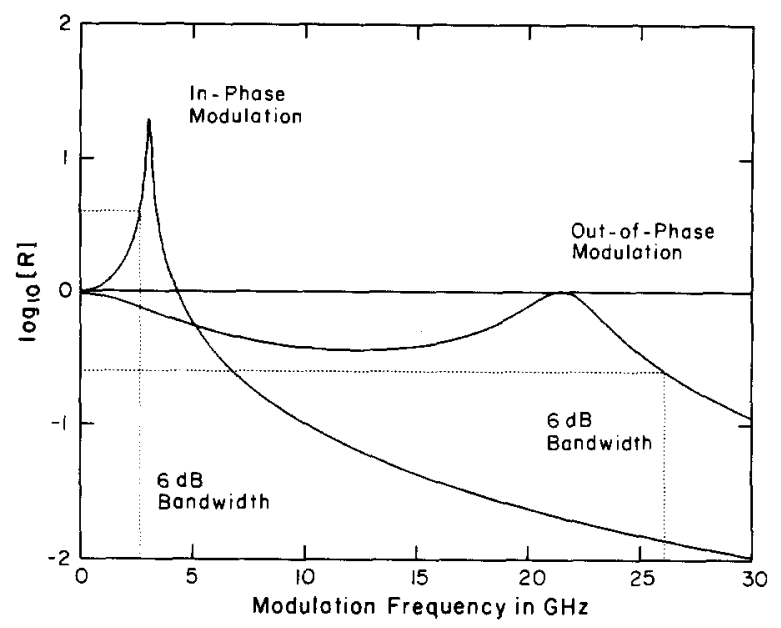

Fig. 1. Response versus modulation frequency for a twin emitter semiconductor laser subjected to infinitesimal modulation, for both in-phase and out-of-phase modulation. The $6 \mathrm{~dB}$ bandwidth is a factor of ten larger for out-of-phase modulation than for inphase modulation.

Table 1

\begin{tabular}{llll}
\hline Parameter & Symbol & Value & Ref. \\
\hline Carrier lifetime & $\tau_{\mathrm{s}}$ & $2.4 \mathrm{~ns}$ & {$[3]$} \\
Photon lifetime & $\tau_{\mathrm{p}}$ & $1.5 \mathrm{ps}$ & {$[3]$} \\
Linewidth enhancement factor & $\alpha$ & 7.5 & {$[3]$} \\
Normalized injection current bias & $p^{(0)}$ & 0.66 & {$[3,8]$} \\
\hline
\end{tabular}

itter array characterized by $\eta_{\mathrm{R}}=0.1, \eta_{1}=0$ and the parameters given in table 1 . These parameters were measured for a gain-guided twin emitter [3] biased at $I=1.7 I_{\mathrm{thr}}$. For in-phase modulation, the response is maximum at the frequency

$f_{\text {res }}=\left(\frac{p^{(0)}}{2 \pi^{2} \tau_{\mathrm{p}} \tau_{\mathrm{s}}}\right)^{1 / 2}=3.0 \mathrm{GHz}$.

The strong frequency dependence of this response, as shown in fig. 1 , is not conductive to the faithful reproduction of signals. If we define the data transmission bandwidth as the frequency at which the response first crosses the $\pm 6 \mathrm{~dB}$ level relative to $R(0)$, such that higher-frequency signals are unacceptably distorted, the bandwidth for in-phase modulation is only $2.6 \mathrm{GHz}$. Furthermore, semiconductor lasers tend to spike at the relaxation oscillation frequency when deeply modulated at a lower frequency [13], if the resonance response is sufficiently large. Such 
behavior would clearly be detrimental for communications applications.

Fig. 1 also shows the response to out-of-phase modulation. The peak response occurs at

$f_{\text {res }}=\eta_{\mathrm{R}} / \pi \tau_{\mathrm{p}}=21 \mathrm{GHz}$,

and there is no trace of a peak at the relaxation oscillation frequency. The previously defined data transmission bandwidth is $26 \mathrm{GHz}$ for out-of-phase modulation, ten times that of in-phase modulation. The average out-of-phase response is somewhat smaller than the average in-phase response, but is undegraded by oscillations at higher frequencies since there are no resonances beyond the bandwidth.

\section{Stability of periodic responses}

The foregoing analysis presupposes the existence of a stable periodic solution to the modulated rate equations. This assumption has not been justified, and proves to be untrue over much of the space spanned by device and modulation parameters. Periodic solutions predicted by the model are of little interest if they are unstable and thus never observed. To proceed, it is necessary to address the question of stability.

We consider only infinitesimal modulation, for which the periodic solutions found should possess the same stability property as the parent equilibrium point. Unfortunately, both equilibrium points are unstable over a large portion of the complex $\eta$ plane. For $\eta_{1} \approx 0$, both equilibrium points are unstable over the range of interest, $0.03<\eta_{R}<0.3$, which corresponds to the modulation frequency range 6 $\mathrm{GHz}<f_{\text {mod }}<60 \mathrm{GHz}$ for the device parameters given in table 1. Using an appreciable $\eta_{\mathrm{I}}$ can stabilize one equilibrium point, but only at the expense of diminishing the mode beating that is necessary for highfrequency modulation.

Fig. 2 is a $\log -\log$ plot of the $\eta_{R}>0$ half of the complex $\eta$ plane. Since the properties of the array vary only slightly from $\eta_{1}=-10^{-5}$ to $\eta_{\mathrm{I}}=10^{-5}$, the $\eta_{\mathrm{I}}<0$ and $\eta_{\mathrm{I}}>0$ quadrants have been joined at $\left|\eta_{\mathrm{I}}\right|=10^{-5}$, which for all practical purposes can be regarded as the real axis. Since $\eta_{\mathrm{R}}<0$, which corresponds to the out-of-phase mode having a lower frequency than the in-phase mode, has not to the best of our knowledge

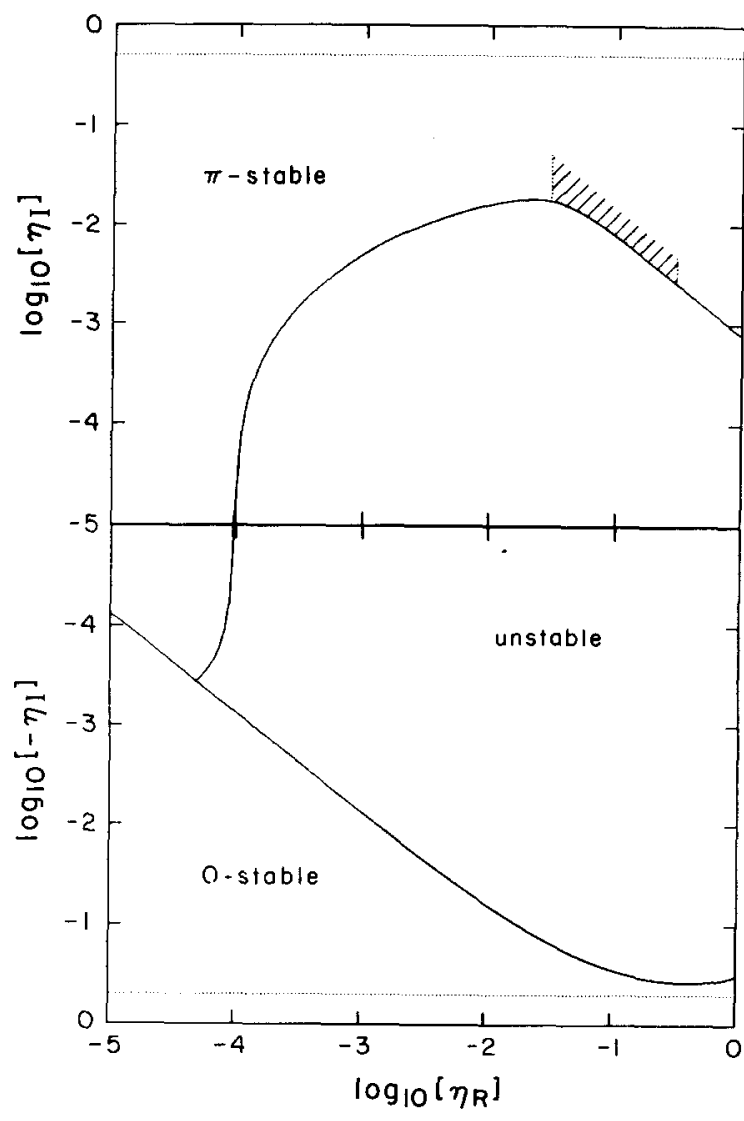

Fig. 2. The $\eta_{\mathrm{R}}>0$ complex $\eta$ half-plane. The $\eta_{1}<0$ and $\eta_{\mathrm{r}}>0$ quadrants are joined at $\left|\eta_{\mathrm{I}}\right|=10^{-5}$. The regions of stability of the $\theta^{(0)}=0$ and $\theta^{(0)}=\pi$ equilibrium points of the unmodulated twin emitter semiconductor laser are bounded by the curved solid lines and the horizontal straight dotted lines. Both equilibrium points are unstable in the central region. The optimal design region is shown hatched.

been experimentally observed, the other half-plane is not considered.

The $\eta_{R}>0$ half-plane is partitioned into three regions according to the linear asymptotic stability of the equilibrium points of a twin emitter with the parameters given in table 1: in-phase stable, out-ofphase stable and neither stable. The boundaries are found by a technique introduced [8] for real $\eta$ and extended [5] to complex $\eta$. They have been verified by numerically integrating the rate equations with no modulation for $\eta$ on either side of a boundary and checking for the loss of stability of the appropriate equilibrium point. They also conform to theoretical predictions [8] for $\eta_{1} \rightarrow 0$. The hatching in fig. 2 rep- 


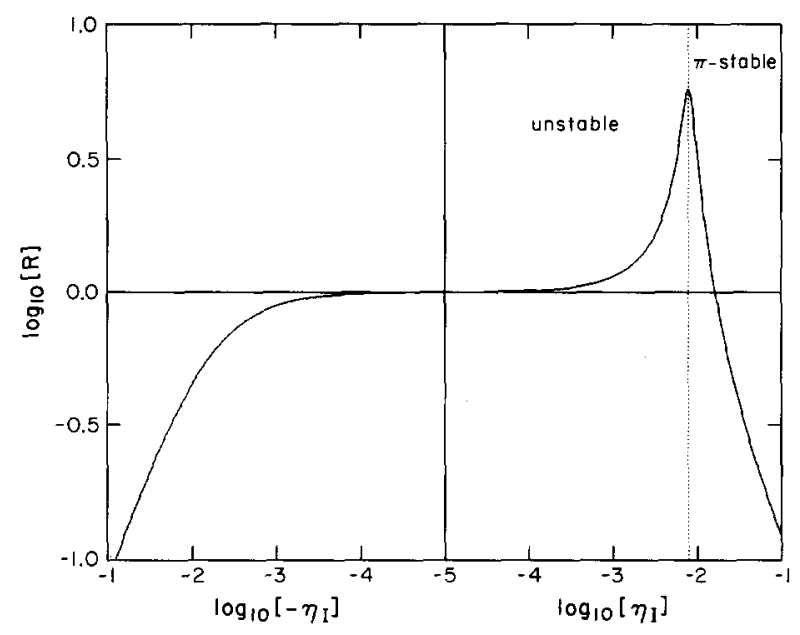

Fig. 3. Response to infinitesimal modulation versus $\eta_{1}$ for $\eta_{R}=0.1$ and $f_{\bmod }=\eta_{\mathrm{R}} / \pi \tau_{\mathrm{p}}=21.2 \mathrm{GHz}$. For $\eta_{\mathrm{I}}<0, \theta^{(0)}=0$ is assumed, while for $\eta_{\mathrm{I}}>0, \theta^{(0)}=\pi$ is assumed. The region to the right of the dotted line is stable to small-signal modulation about the $\theta^{(0)}=\pi$ equilibrium point.

resents the optimal design region, formed by the intersection of the $\eta_{\mathrm{R}}$ range of interest and the $\eta_{\mathrm{I}}$ that gives a large but stable response to modulation, as discussed below.

Fig. 3 shows the response to small-signal out-ofphase modulation at the resonance frequency $2 \eta_{\mathrm{R}}$ of a twin emitter with the parameters given in table 1 , as a function of $\eta_{1}$, for $\eta_{R}=0.1$. Because of the stability properties of the equilibrium points, oscillation about the in-phase equilibrium point for $\eta_{1}<0$ and the out-of-phase equilibrium point for $\eta_{1}>0$ was assumed. In the $\eta_{R}$ range of interest, the boundary between the out-of-phase stability region and the instability region is approximately given by

$\eta_{\mathrm{I}, \text { bound }}=\alpha p^{(0)} / 4 T \eta_{\mathrm{R}}$.

Moreover,

$R\left(\Omega=2 \eta_{\mathrm{R}}, \eta_{\mathrm{I}}=2 \eta_{\mathrm{I}, \text { bound }}\right)=1$.

Thus a large but stable response may be had by choosing $\eta_{\mathrm{I} \text {, bound }}<\eta_{\mathrm{I}}<2 \eta_{\mathrm{I}, \text { bound }}$. For larger $\eta_{\mathrm{I}}$ the response diminishes rapidly.

\section{Summary and conclusions}

Semiconductor lasers are indispensable to high- speed optical communication technology. The relaxation oscillation frequency, a modulation limit for a single-mode laser, can be exceeded by modulating at a beat frequency of a multi-mode laser. Unfortunately, the frequency associated with the spacing of the longitudinal modes of semiconductor lasers is typically beyond the capabilities of present semiconductor laser driver technology. The possibility of modulating a twin-emitter array at the beat frequency of the lateral modes has been presented. This frequency is typically intermediate to the relaxation oscillation and longitudinal mode spacing frequencies.

A rate equation model of a twin-emitter array was introduced and used to find the response of the optical output of the array to small sinusoidal modulations of the injection current. In-phase modulation of the emitters was found to be ineffectual, while modulating the emitters $180^{\circ}$ out of phase extended the responsivity well beyond the relaxation oscillation frequency, to approximately the frequency associated with the rate of photon exchange between the coupled emitters.

It was also shown that high responsivity could be obtained by choosing $\eta_{\mathrm{I} \text {,bound }}<\eta_{\mathrm{I}}<2 \eta_{\mathrm{I} \text {,bound }}$, which produces a stable periodic orbit about the out-ofphase equilibrium point in the small-signal limit.

In conclusion, it appears that twin-emitter semiconductor laser phased arrays can be modulated at frequencies beyond the relaxation oscillation frequency limit, and may be useful as light sources in high-speed optical communications. Furthermore, it is possible in principle to extend the present analysis to larger arrays to achieve higher optical powers simultaneous with high modulation rates, which is the topic of our current studies.

\section{Acknowledgement}

The authors wish to acknowledge the support of the National Science Foundation, the Air Force Office of Scientific Research and the Army Research Office through the URI program. 


\section{References}

[1] K.Y. Lau, Appl. Phys. Lett. 52 (1988) 2214.

[2] R.K. DeFreez, N. Yu, D.J. Bossert, M. Felisky, G.A. Wilson, R.A. Elliott, H.G. Winful, G.A. Evans, N.W. Carlson and R. Amantea, in: Proc. Opt. Soc. Am. Topical Meeting on Nonlinear Dynamics in Optical Systems (Afton, OK, USA, 1990), Paper ThAl.

[3] N. Yu, Ph.D. Thesis, Oregon Graduate Institute of Science and Technology, Beaverton, OR, USA (1990).

[4] R.K. DeFreez, D.J. Bossert, N. Yu, J.M. Hunt, H. Ximen, R.A. Elliott, N.W. Carlson, M. Lurie, G.A. Evans, J.M. Hammer, D.P. Bour, S.L. Palfrey, R. Amantea, H.G. Winful and S.S. Wang, IEEE Photonics Tech. Lett. 1 (1989) 209.

[5] G.A. Wilson, Ph.D. Thesis, unpublished.

[6] K.A. Shore and T.E. Rozzi, Optical nonlinearities and instabilities in semiconductors (Academic Press, New York, 1988) Ch. 17.
[7] K.A. Shore and T.E. Rozzi, Opt. Quantum Electron. 15 (1983) 497.

[8] H.G. Winful and S.S. Wang, Appl. Phys. Lett. 53 (1988) 1894.

[9] J.K. Butler, D.E. Ackley and M. Ettenberg, IEEE J. Quantum Electron. QE-21 (1985) 458.

[10] G.P. Agarwal, in: Proc. Opt. Soc. Am. Topical Meeting on Nonlinear Dynamics in Optical Systems (Afton, OK, USA, 1990), Paper MA2.

[11] H. Kressel and J.K. Butler, Semiconductor lasers and heterojunction LEDs (Academic Press, New York, 1977) section 17.5.

[12] R.S. Tucker, J. Lightwave Technol. LT-3 (1985) 1180.

[13] E. Hemery, L. Chusseau and J.-M. Lourtioz, IEEE J. Quantum Electron. QE-26 (1990) 633. 